01

\title{
Униполярная модель отрицательного коронного разряда: сравнение рассчитанных и экспериментальных вольт-амперных характеристик в системе электродов сфрера-плоскость
}

\author{
(C) Н.В. Мельникова, А.В. Самусенко, Ю.Ф. Сафронова
}

Санкт-Петербургский государственный университет, 199034 Санкт-Петербург, Россия

e-mail: a.samusenko@spbu.ru

\section{(Поступило в Редакцию 26 сентября 2016 г.)}

\begin{abstract}
Компьютерное моделирование коронного разряда с учетом процессов в чехле требует значительных ресурсов памяти и расчетного времени. В связи с этим актуальным является вопрос поиска и усовершенствования упрощенных моделей, в которых описание происходящих в объеме чехла коронного разряда процессов сводится к граничному условию на поверхности активного электрода. Рассматривается недавно предложенная униполярная (учитывающая носители заряда только одного знака) модель, в которой граничное условие на коронирующем электроде описывает скорость изменения плотности потока электронов из чехла. Рассчитанные при помощи этой модели вольт-амперные характеристики сопоставляются с экспериментальными данными в диапазоне межэлектродных расстояний от нескольких $\mathrm{mm}$ до нескольких $\mathrm{cm}$ для выявления области применимости модели. Модель показывает хорошее соответствие с экспериментом для межэлектродных расстояний больше $1 \mathrm{~cm}$.
\end{abstract}

DOI: $10.21883 / J T F .2017 .08 .44716 .2043$

\section{Введение}

Электрический ветер - течение газа, вызванное коронным разрядом [1]. Это явление находит применение в промышленных электрофильтрах [1], охлаждающих устройствах [2-5], прототипах летательных аппаратов $[6,7]$, в управлении профилем обтекания $[8,9]$.

Компьютерное моделирование коронного разряда на основе полной системы уравнений с рассмотрением ионизационных процессов в чехле крайне ресурсоемко. Поэтому разрабатываются упрощенные модели, основанные на униполярном приближении: в объеме рассматривается только внешняя зона разряда, в которой присутствуют заряженные частицы одного знака (соответствующего полярности активного электрода) и нет ионизации [10]. Чехол коронного разряда в таких моделях описывается посредством граничного условия на поверхности коронирующего электрода. Традиционные модели обычно используют условие на постоянство напряженности электрического поля после зажигания разряда $E=E_{0}$ (значение пороговой напряженности $E_{0}$ для цилиндрических электродов можно найти по формуле Пика [1], для других геометрий требуется его подбирать) либо определяют плотность потока ионов $j$ как некоторую функцию напряженности поля $j=f(E)$. В качестве функции $f(E)$ берут, как правило, простую модельную зависимость, например линейную, коэффициенты которой подбираются для соответствия экспериментальным данным. Такого рода модели в общем случае требуют переопределения коэффициентов при любом изменении геометрии. Это приводит к необходимости получать экспериментальные данные при каждой модификации геометрии, что ограничивает общность модели и снижает ее ценность.
В [11] предложено граничное условие для униполярной модели в виде скорости изменения плотности потока электронов $j_{e}$ :

$$
\frac{\partial j_{e}}{\partial t}=j_{e} \frac{\gamma(\exp M-1)-1}{\tau}
$$

где $\gamma$ - коэффициент вторичной эмиссии с поверхности электрода, $M$ - число ионизационных столкновений, $\tau$ - время пролета положительных ионов сквозь чехол коронного разряда. Следует отметить, что $\tau$ по порядку величины не превышает долей миллисекунд, и в стационарном режиме, в котором рассчитываются значения токов для вольт-амперной характеристики, значение данного параметра не влияет на результат расчета.

Условие (1) в качестве параметров содержит коэффициент вторичной эмиссии $\gamma$, определяющийся материалом активного электрода и состоянием его поверхности. Этот коэффициент не меняется при изменении взаимного расположения электродов, что является преимуществом модели - она может быть использована для оптимизации конструкции устройств на основе электрического ветра. В [12] было проведено сравнение результатов моделирования и эксперимента (вольт-амперные характеристики, поля скоростей) в двух геометрических конфигурациях: в системах сфера-плоскость и игла-плоскость, которое продемонстрировало хорошее соответствие между моделью и экспериментом.

В униполярной модели объемные процессы в чехле коронного разряда заменяются граничным условием, структура чехла при этом не рассматривается. Очевидно, подобное упрощение может быть применимо только в том случае, когда межэлектродное расстояние существенно превышает характерный размер чехла. 


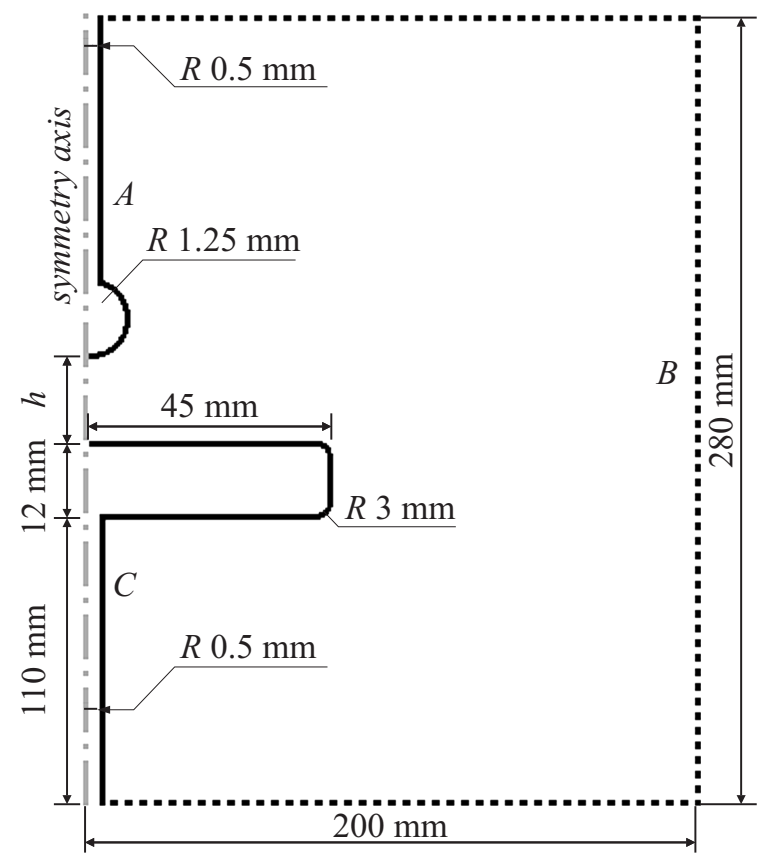

Рис. 1. Схема расчетной модели.

Поэтому представляет интерес вопрос о том, в каком диапазоне межэлектродных расстояний рассматриваемая упрощенная модель применима. Этот вопрос далее будет рассмотрен для случая коронного разряда отрицательной полярности в системе электродов сфера-плоскость.

\section{Эксперимент}

Исследование проводилось в системе электродов сфера-плоскость (рис. 1) при варьировании межэлектродного расстояния $h$.

В качестве высоковольтного электрода использовалась стальная сфера радиуса $1.25 \mathrm{~mm}$, закрепленная на тонком металлическом цилиндре диаметром $1 \mathrm{~mm} \mathrm{c}$ помощью магнитных сил. Металлический цилиндр выполнял функции провода, подводящего высокий потенциал. Заземленный электрод представляет собой медный цилиндр высотой $12 \mathrm{~mm}$ и диаметром $9 \mathrm{~mm}$.

Напряжения и токи регистрировались при помощи двухканального АЦП L-Card E-502. Измерение тока осуществлялось путем регистрации напряжения на сопротивлении $1 \mathrm{k} \Omega$, последовательно включенным в цепь источник - разрядный промежуток.

Подача высокого напряжения отрицательной полярности осуществлялась при помощи источника постоянного напряжения ИВН-50.

\section{Моделирование}

Система уравнений (2)-(5) аналогична рассмотренной в [12] и содержит уравнение Пуассона (2), уравнения Нернста-Планка для электронов и отрицательных ионов (4), (5), а также уравнение для интегрирования коэффициента ионизации $\alpha(E)$ вдоль силовых линий электрического поля (3):

$$
\left\{\begin{array}{l}
\varepsilon_{0} \Delta \varphi=-e\left(n_{e}+n_{i}\right) \quad(\mathbf{E}=-\nabla \varphi), \\
(\mathbf{E} / E \nabla) M=\alpha(E), \\
\frac{\partial n_{e}}{\partial t}+\operatorname{div}\left[-D_{e} \nabla n_{e}-n_{e} \mu_{e} \mathbf{E}\right]=-v_{\mathrm{att}}(E) n_{e}, \\
\frac{\partial n_{i}}{\partial t}+\operatorname{div}\left[-D_{i} \nabla n_{i}-n_{i} \mu_{i} \mathbf{E}\right]=+v_{\mathrm{att}}(E) n_{e} .
\end{array}\right.
$$

Искомыми функциями в системе уравнений (2)-(5) являются распределения электрического потенциала $\varphi$, концентрации электронов $n_{e}$, концентрации ионов $n_{i}$. Также рассчитывается распределение числа ионизационных соударений $M$, использующееся в граничном условии (1). $D_{e}, D_{i}$ - коэффициенты диффузии, $\mu_{e}, \mu_{i}-$ подвижности носителей заряда. Индексы $e$ и $i$ относятся к электронам и ионам соответственно. $v_{\text {att }}-$ коэффициент прилипания электронов к нейтральным молекулам, $e-$ элементарный заряд, $\varepsilon_{0}$ - диэлектрическая постоянная.

Зависимость коэффициента ионизации $\alpha$ от напряженности поля $E$ задана в соответствии с интерполирующей функцией $\alpha(E)=A \exp (-B / E)$ по данным [13], где $A$ и $B-$ постоянные. Подвижность электронов $\mu_{e}(E)$, коэффициент диффузии электронов $D_{e}(E)$, частота прилипания $v_{\text {att }}(E)$ заданы как аппроксимации экспериментальных данных [14,15]. Выбранное значение подвижности ионов $\mu_{i}=2.6 \cdot 10^{-4} \mathrm{~m}^{2} /(\mathrm{V} \cdot \mathrm{s})$, согласуется с данными [1] и измерениями подвижности ионов по вольт-амперным характеристикам короны на больших межэлектродных расстояниях [16]. Исходя из соотношения Эйнштейна, коэффициент диффузии ионов задан равным $D_{i}=\mu_{i} k_{\mathrm{B}} T=8 \cdot 10^{-6} \mathrm{~m}^{2} / \mathrm{s}$, где $k_{\mathrm{B}}-$ постоянная Больцмана, $T=293 \mathrm{~K}$. Коэффициент вторичной эмиссии взят равным $\gamma=4 \cdot 10^{-4}$.

Граничные условия приведены в таблице. $U-$ напряжение на высоковольтном электроде, индекс n обозначает нормаль к границе.

Система уравнений (2)-(5) решается как переходная задача, однако расчет ведется при подаче постоянного напряжения до выхода на стационар, при котором и вычисляется значение тока, соответствующее данному напряжению.

Граничные условия

\begin{tabular}{c|c|c|c}
\hline $\begin{array}{c}\text { Уравне- } \\
\text { ние }\end{array}$ & $\begin{array}{c}A-\text { высоковольт- } \\
\text { ный электрод }\end{array}$ & $\begin{array}{c}B-\text { внешняя } \\
\text { граница модели }\end{array}$ & $\begin{array}{c}C-\text { заземлен- } \\
\text { ный электрод }\end{array}$ \\
\hline$(2)$ & $\varphi=-U$ & $\frac{\partial \varphi}{\partial n}=0$ & $\varphi=0$ \\
$(3)$ & - & - & $M=0$ \\
$(4)$ & $(1)$ & $\left(j_{e}\right)_{n}=0$ & - \\
$(5)$ & $j_{i}=0$ & $\left(j_{i}\right)_{n}=0$ & -
\end{tabular}




\section{Результаты}

Как видно из рис. 2, расчетные вольт-амперные характеристики хорошо соотносятся с экспериментальными при межэлектродных расстояниях, превышающих сантиметр, а при меньших - расчет систематически занижает значение токов.

И расчетные, и экспериментальные вольт-амперные характеристики выше порога зажигания хорошо аппроксимируются квадратичной функцией вида

$$
I(U)=K U\left(U-U_{0}\right) .
$$

Вольт-амперная характеристика в таком случае определяется двумя параметрами: порогом зажигания $U_{0}$ и коэффициентом $K$. Эти параметры могут быть рассчитаны путем линейной аппроксимации отношения $I / U$, как функции напряжения $U$. На рис. 3,4 приведены расчетные и экспериментальные зависимости $U_{0}$ и $K$ от межэлектродного расстояния. Расчетные значения $U_{0}$ находятся в удовлетворительном соответствии с экспериментом во всем диапазоне межэлектродных расстояний, отклонение не превышает 7\%. Расчетные значе-

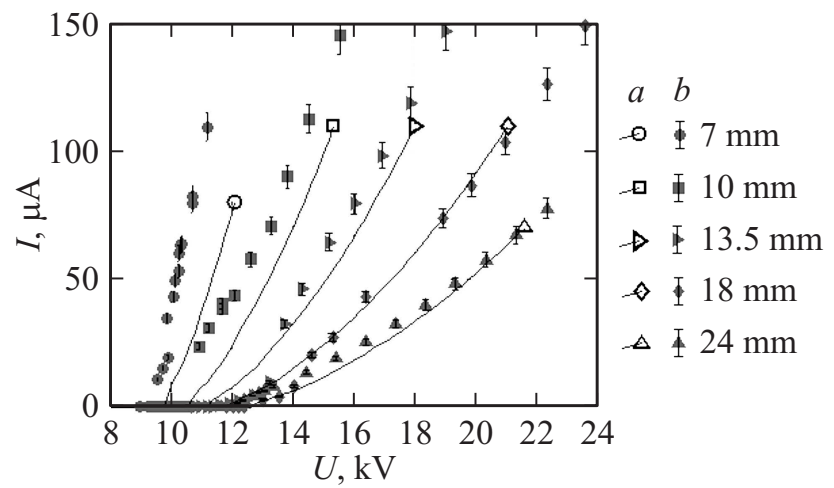

Рис. 2. Расчетные и экспериментальные вольт-амперные характристики при разных межэлектродных расстояниях $h ; a-$ расчет, $b-$ эксперимент.

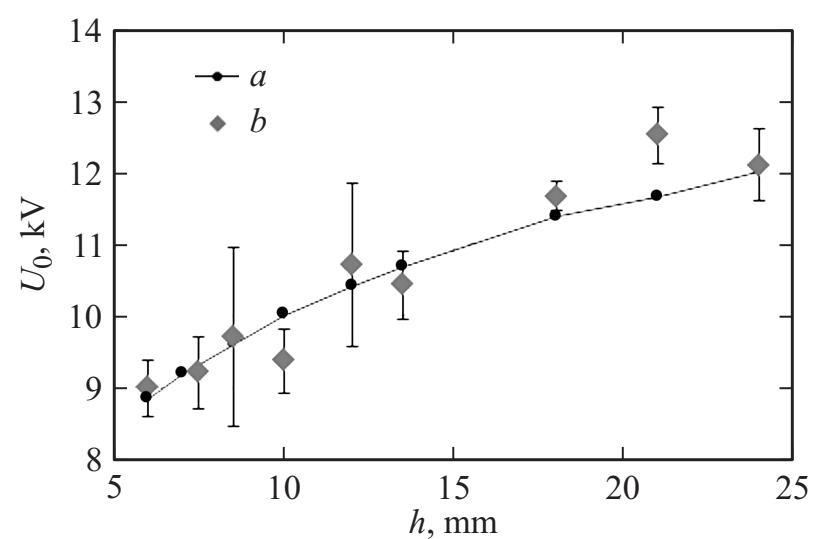

Рис. 3. Зависимость порога возникновения коронного разряда $U_{0}$ от межэлектродного расстояния $h$. Сопоставление экспериментальных и расчетных значений; $a-$ расчет, $b-$ эксперимент.

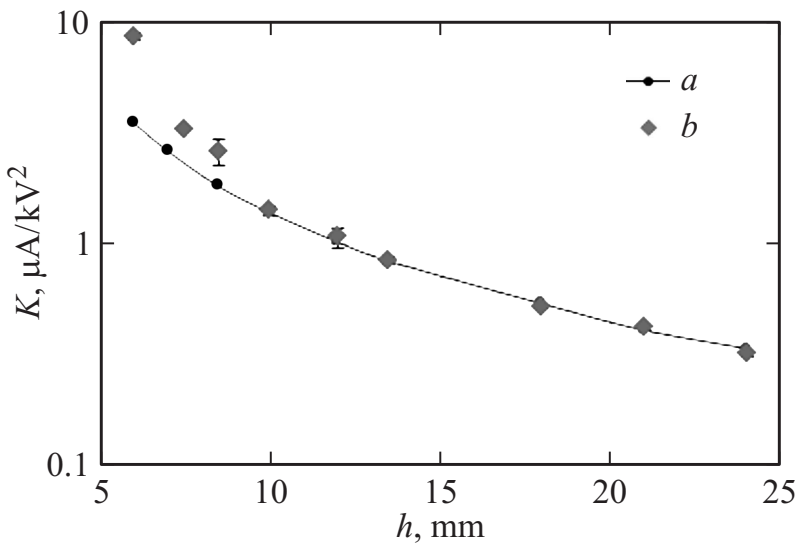

Рис. 4. Зависимость коэффициента $K$ (см. формулу (6)) от межэлектродного расстояния $h$. Сопоставление экспериментальных и расчетных значений; $a-$ расчет, $b-$ эксперимент.

ния $K$ находятся в хорошем согласии с экспериментом для межэлектродных расстояний, превышающих $1 \mathrm{~cm}$ (отклонение не превышает 7\%). При расстояниях менее $1 \mathrm{~cm}$ различие быстро увеличивается с уменьшением межэлектродного расстояния: $30 \%$ при $h=1 \mathrm{~cm}, 50 \%$ при $h=7 \mathrm{~mm}, 80 \%$ при $h=6 \mathrm{~mm}$.

Тот факт, что сильное расхождение наблюдается именно по коэффициенту $K$, а не по порогу зажигания, говорит о том, что расхождение связано именно с описанием коронного разряда, а не процессов, предшествующих его появлению. Можно предположить, что прежде всего причиной расхождения является отсутствие в модели облака положительных ионов в чехле коронного разряда. Наличие этой компоненты в структуре объемного заряда ослабляет экранирование поля доминирующим в короне отрицательным объемным зарядом и, как следствие, делает возможным больший уровень тока.

\section{Выводы}

Рассматриваемая униполярная модель отрицательной короны с граничным условием (1) в виде скорости выхода потока электронов с коронирующего электрода в качестве входных параметров включает известные физические величины, поддающиеся независимому измерению: коэффициент ионизации, подвижности отрицательных ионов и электронов, коэффициенты диффузии, коэффициент вторичной ион-электронной эмиссии. Такая модель не требует дополнительных подгоночных параметров при изменении взаимного расположения электродов.

Сопоставление экспериментальных и расчетных вольт-амперных характеристик для системы электродов сфера-плоскость показало, что эта модель может применяться для расчетов при межэлектродных расстояниях больше $1 \mathrm{~cm}$. Для межэлектродных расстояний порядка единиц $\mathrm{mm}$ модель систематически завышает величину тока короны. 


\section{Список литературы}

[1] Верещагин И.П. Коронный разряд в аппаратах электронно-ионной технологии. М.: Энергоатомиздат, 1985. 160 с.

[2] Yang $F$. et al. // Proc. of the International Symposium on High Voltage Engineering. Delft, Netherlands, 2003. P. 1-4.

[3] Go D.B., Garimella S.V., Fisher T.S., Mongia R.K. // J. Appl. Phys. 2007. Vol. 102. N 5. P. 053302.

[4] Chen I.Y., Guo M.-Z., Yang K.-S., Wang C.-C. // Int. J. Heat Mass Tran. 2013. Vol. 57. N 1. P. 285-291.

[5] Ong A.O., Abramson A.R., Tien N.C. // J. Heat Mass Tran. 2014. Vol. 136. N 6. P. 061703.

[6] Siswanto W.A., Ngui K. // Aust. J. Basic. Appl. Sci. 2011. Vol. 5. N 9. P. 1433-1438.

[7] Ianconescu R., Sohar D., Mudrik M. // J. Electrostat. 2011. Vol. 69. N 6. P. 512-521.

[8] Léger L., Moreau E., Artana G., Touchard G. // J. Electrostat. 2001. Vol. 51-52. N 1-4. P. 300-306.

[9] Léger L., Moreau E., Touchard G. // J. Electrostat. 2006. Vol. 64. N 3-4. P. 215-225.

[10] Adamiak K. // J. Electrostat. 2013. Vol. 71. P. 673-680.

[11] Samusenko A., Stishkov Yu., Zhidkova P. // I.J.PEST. 2015. Vol. 9. N 1. P. 24-28.

[12] Ашихмин И.А., Самусенко А.В., Стишков Ю.К., Яковлев В.В. // ЖТФ. 2015. Т. 85. Вып. 11. С. 65-72.

[13] Райзер Ю.П. Физика газового разряда. Долгопрудный: Интеллект, 2009. 736 с.

[14] Dutton J. // J. Phys. Chem. Ref. Data. 1975. Vol. 4. N 3. P. $577-856$.

[15] Gallagher J.W., Beaty E.C., Dutton J., Pitchford L.C. // J. Phys. Chem. Ref. Data. 1983. Vol. 12. N 1. P. 109-152.

[16] Moreau E., Benard N., Lan-Sun-Luk J.-D., Chabriat J.-P. // J. Phys. D: Appl. Phys. 2013. Vol. 46. P. 475204. 